\author{
VIRAGO VALOROSA O "MARFISA BIZARRA"? \\ LA DONNA GUERRIERA NE LA PAZZIA D'ISABELLA (1611) \\ DI FLAMINIO SCALA \\ E NE Lo SCHIAVETTO (1612) Di Giovan BATTISTA ANDREINI
}

\title{
VALENTINA DENZEL
}

Riassunto: In questo articolo confrontiamo la messa in scena dei personaggi femminili delle due commedie La pazzia di Isabella (1611) di Flaminio Scala e Lo Schiavetto (1612) di Giovan Battista Andreini. Le protagoniste della commedia di Scala, Isabella e Flaminia, rievocano per la loro presa d'armi le guerriere ariostesche, Marfisa e Bradamante. Per vendicare l'infedeltà maschile e per proteggere i loro interessi, Flaminia ed Isabella ricorrono alla violenza. I loro atti non vengono però giustiziati, ma servono invece a ristabilire l'ordine. In effetti, La pazzia d'Isabella finisce con un matrimonio doppio, celebrato tra le protagoniste e i loro amanti pentiti. Lo Schiavetto rafforza invece la gerarchia tradizionale tra i sessi, concedendo ai personaggi maschili un ruolo più importante. L'immagine della donna guerriera viene parodiata, perché la commedia di Andreini allude, tra l'altro, al poema epico Marfisa bizarra (1531) di Giovan Battista Dragoncino, che mette in ridicolo la virago ariostesca.

Il destino del paladino Orlando, l'eroe eponimo dell'Orlando furioso (1532) di Ludovico Ariosto, è stato adattato frequentemente nei canovacci della Commedia dell'arte. L'Orlando Furioso (1622) di Basilio Locatelli e La grande pazzia d'Orlando, commedia di cui si ignorano l'autore e la data, sono due esempi della ripresa della materia ariostesca nella scenografia dell'Arte.

Il drammaturgo Flaminio Scala (1547-1624), attore e direttore della compagnia dei Confidenti, s'ispirò ugualmente all'avventura amorosa del paladino cristiano che per amore perse la ragione. Non essendo ricambiato dalla principessa Angelica, la quale gli preferisce un semplice soldato saraceno, Orlando diventa pazzo. Accecato dalla gelosia e dal dolore, il celebre paladino si trasforma in personaggio feroce che minaccia ogni essere vivente ${ }^{1}$.

1 Ariosto, Orlando furioso, XIX, 28-29; XXIV, 4-5. D'ora in avanti, i riferimenti a quest'opera saranno inseriti nel testo usando la sigla O.f. e il numero del canto. 
La sua commedia La pazzia d'Isabella, pubblicata nel 1611, ma facente parte delle prime opere della prima generazione della commedia dell'arte $^{2}$, è contenuta nella raccolta Il teatro delle favole rappresentative (1611). In questa commedia, Scala fa subire la follia d'amore di Orlando al personaggio di Isabella. Le analogie tra La pazzia d'Isabella e l'Orlando furioso non si limitano però a questo. La commedia di Scala mette ugualmente in scena due personaggi femminili che ricorrono alle armi: Isabella e la sua rivale Flaminia. Tale aspetto ricorda le due famose donne guerriere dell'epopea ariostesca: Marfisa e Bradamante. Anche se Isabella e Flaminia non sono delle virago comparabili con queste due guerriere, giacché non indossano le armi come fanno Marfisa e Bradamante, ma si trasformano solo per un attimo in "amazzoni", il mettere in scena una certa violenza "femminile" resta un punto in comune tra questi quattro personaggi ${ }^{3}$.

$\mathrm{Ne}$ La pazzia d'Isabella, le due protagoniste proteggono i loro interessi nei confronti di due personaggi maschili grazie all'uso della violenza. Siccome Scala rappresenta raramente delle donne armate, questo fatto ci sembra degno di attenzione. Nella maggior parte dei canovacci, le protagoniste si difendono dalla supremazia maschile tramite una posizione superiore situata al disopra della scena, spesso una finestra. Jane Tylus, ricercatrice americana, afferma che la maggior parte delle commedie di Scala - 109 su un totale di 120 - mostra le attrici affacciate alla finestra ${ }^{4}$. Da questo punto superiore, i personaggi femminili non solo controllano le azioni che si svolgono in piazza o per istrada, ma possono anche intervenire e cambiare il corso della trama grazie alla loro posizione che domina la scena. La commedia Le disgrazie di Flavio, contenuta ugualmente nella raccolta Il teatro delle favole rappresentative (1611), mette per esempio in scena la punizione dell'amante infedele Flavio. Isabella, credendo che Flavio ami Flaminia, "vien dalla fenestra fuora, salta addosso a Flavio, gli dà de' pugni, senza pur lasciarli dire una parola" (atto II, p. 358). Inoltre, Isabella "alla fenestra saluta amorosamente il Capitano, per far dispetto a Flavio” (atto II,

2 Tylus, "Women at the Windows", 327.

3 Anche le due commedie L'Orlando Furioso (1622) e La grande pazzia d'Orlando mettono in scena Marfisa e Bradamante. Bisogna però dire che nella prima commedia, le due donne guerriere appaiono soltanto sull'indice dei personaggi senza essere menzionate nel canovaccio. La seconda, pure mettendo sull'indice i nomi di tutte e due le virago, descrive soltanto il personaggio di Bradamante, omettendo quello di Marfisa.

4 Tylus, "Women at the Windows", 327.

5 Tylus, "Women at the Windows", 328. 
p. 359). Come dimostra Jane Tylus, l'introduzione dell'attrice sulla scena è un'innovazione scenografica rispetto alle convenzioni teatrali precedentis. La posizione superiore dell'attrice affacciata alla finestra incarna però anche la traiettoria che va da un personaggio passivo ad una figura femminile attiva, senza che la sua autonomia trasgredisca le convenzioni sociali.

The theatre of windows introduces female spectatorship onto the stage in such a way as to allow the woman to transform traditional passivity into manipulative action. On the other hand, in Scala's Teatro, the woman who renders herself completely visible and therefore vulnerable, ever a part of a public space she wishes to dominate through her sexuality and charm, is ultimately no match for the real actress, whose deployment of the boundaries between public and private spaces make her superior to the shadowy and finally unacceptable figure of the courtesan. ${ }^{6}$

Ora, La pazzia d'Isabella differisce da questa tattica di controllo femminile. Invece di sfruttare il punto strategico della finestra, Isabella e Flaminia preferiscono l'uso delle armi. In questo articolo vorremmo analizzare questa particolarità della commedia di Scala, tenendo anche conto del fenomeno della Querelle des femmes. Questa disputa letteraria e iconografica, si colloca nell'Europa del Quattrocento fino al Settecento, pur continuando come fenomeno endemico nei secoli successivi. L'argomento fondamentale della Querelle des femmes, chiamata anche "Le polemiche sul sesso femminile", è il dibattito sul pregio e il vizio delle donne e sulla loro posizione politico-sociale 7 .

L'insolita presa delle armi da parte di Isabella e di Flaminia nella commedia di Scala, sembra essere un'allusione alle due donne guerriere ariostesche. Quest'influenza dell'epopea dell'Ariosto sulla scenografia de La pazzia d'Isabella è suggerita dalle circostanze nelle quali Isabella e Flaminia fanno uso delle armi.

La prima a fare ricorso ad un coltello è Isabella. Il suo atto di violenza non si dirige contro il suo amante infedele Orazio, né contro la sua rivale Flaminia, alla quale Orazio ha fatto promessa di matrimonio, ma contro Flavio che diffama il comportamento vergognoso di Orazio.

Pantalone viene esortando Flavio a placarsi con Orazio, il qual dice che non farà mai pace seco, sin tanto ch'egli non sposa Isabella come è di obligo suo, e di volerlo far conoscere che è un grandissimo traditore.

\footnotetext{
6 Tylus, "Women at the Windows", 337.

7 Zimmermann, "Vom Streit der Geschlechter", 17.
} 
Isabella, avendo un coltello allato, se li accosta, dicendoli che mente, e li dà due o tre ferite. Flavio cade in terra versando il sangue; in quello Orazio arriva. Isabella, abbracciandolo e dicendoli d'aver fatte le sue vendette, lo conduce in casa. (Scala, La pazzia d'Isabella, II, p. 392.)

Se è vero che l'Orlando furioso non fornisce un modello preciso a questa scena - visto che le circostanze, nelle quali Bradamante è confrontata all'infedeltà di Ruggiero ${ }^{8}$, differiscono da quelle messe in scena da Scala il legame tra l'opera ariostesca e La pazzia d'Isabella si fonda sulla volontà femminile di proteggere l'amato e sull'intervento di un terzo personaggio. Il primo punto in comune è sicuramente lo zelo con il quale Isabella e Bradamante cercano di proteggere i loro amanti reciproci. I canti IV e VII dell' Orlando furioso descrivono la liberazione di Ruggiero, l'amante e futuro sposo di Bradamante, dal castello incantato del mago Atlante e dall'isola d'Alcina grazie alla valorosa guerriera. Le prodezze della virago si manifestano soprattutto nel canto IV nel quale Bradamante è descritta come "sola ... redentrice" di Ruggiero (O.f., IV, xlii, 2).

Inoltre, le analogie tra Isabella e Bradamante sono evidenziate tramite un terzo personaggio: Flaminia, la rivale di Isabella e Melissa, la maga benevola dell' Orlando furioso. Dopo che Isabella ha aggredito Flavio, Flaminia si rivolge a lei chiamandola "guerriera". Parlando con Orazio, l'amante infedele di Isabella, Flaminia gli chiede con tono ironico "quand'egli farà le nozze con quella sua guerriera, che ha saputo così ben ferire Flavio" (Scala, La pazzia d'Isabella, II, p. 393.). Questo sopranome potrebbe alludere a Bradamante. La rappresentazione di Isabella come "guerriera" si fa però in un contesto ironico e dispregiativo. Ciononostante, l'atteggiamento critico di Flaminia verso il gesto violento di Isabella sarà attenuato nel proseguimento della trama. Flaminia diventerà lei stessa una "guerriera", facendo anche ricorso alle armi. Nello svolgimento della commedia, Flaminia decide di ammazzare Orazio a causa della sua infedeltà verso Isabella e della falsa promessa di matrimonio fatta a Flaminia. Il tentativo d'omicidio non è rappresentato sulla scena ma è raccontato dalla vittima stessa, Orazio.

Orazio querelandosi di Flaminia, che sotto false lusinghe l'abbia condotto in casa e poi assaltatolo con armi per ucciderlo. Ella: che li dispiace

8 Il primo episodio narra il soggiorno di Ruggiero sull'isola d'Alcina (canto VII). La maga Melissa libera Ruggiero dalla lussuria e dalla mollezza, usando l'anello magico di Bradamante. Il secondo episodio racconta la gelosia di Bradamante verso la guerriera Marfisa, che considera erroneamente l'amante di Ruggiero. Bradamante decide di uccidere Ruggiero e la sua rivale (canto XXXVI). 
non averli possuto levar la vita, essend'egli il capo di tutti i traditori, et avvedersi com'era ben cieca a credere alle parole d'uno che aveva e voleva tradir colei che li aveva data la libertà, l'onore, le ricchezze e se stessa ... (Scala, La pazzia d'Isabella, III, pp. 392-393)

Questo atto violento commesso da Flaminia ricorda per vari aspetti l'intervento della maga Melissa sull' isola d'Alcina, narrato nel settimo canto dell' Orlando furioso. Ruggiero arriva dopo varie peripezie sull'isola della maga Alcina, della quale s'innamora. Alcina, lasciva e corrotta, si presenta a Ruggiero sotto un aspetto attraente e giovanile, nascondendo per magia la sua persona decrepita. Per salvare l'unione tra Bradamante e Ruggiero, che fonderanno la casa d'Este (O.f., III, xvii), la maga Melissa decide d'intervenire e di trarre Ruggiero dal suo errore. Melissa si presenta a Ruggiero, criticando il suo comportamento lussurioso e indegno. Il discorso della maga dimostra una certa analogia con quello di Flaminia, che nella commedia di Scala evocava "la libertà, l'onore, le ricchezze e se stessa" che Isabella aveva donato ad Orazio. Melissa ricorda a Ruggiero ugualmente i sacrifici che Bradamante ha fatto per il benessere del paladino.

Quella donna gentil [Bradamante] che t'ama tanto, quella che del tuo amor degna sarebbe, a cui, se non ti scorda, tu sai quanto tua libertà, da lei servata, debbe.

(O.f., VII, lxviii, 1-4)

Melissa e Flaminia intervengono perciò tutte e due per punire l'infedeltà maschile, dimostrando così una certa lealtà verso un altro personaggio femminile, l'amata tradita?.

Un altro legame unisce l'episodio ariostesco dell'isola d'Alcina con la commedia di Scala: Flaminia e Melissa usano la stessa tattica per affrontare gli amanti infedeli. Tutte e due dissimulano le loro intenzioni di punire i traditori. Flaminia finge di ricambiare l'amore di Orazio. Melissa invece

9 Bisogna però aggiungere che la ragione per la quale Flaminia decide di uccidere Orazio è in primo luogo dovuta ad un atto di vendetta. Prima di aggredire Orazio, Flaminia dichiara "di volerlo [Flavio] vendicare, se non contra Isabella, almeno contra Orazio “(Scala 393). Il desiderio di punire l'attentato d'omicidio contro Flavio deriva dal fatto che, prima di cedere alle lusinghe di Orazio, Flaminia intratteneva una relazione amorosa con Flavio. Nel corso della commedia, Flaminia giustifica però il suo atto violento come gesto di lealtà nei riguardi di Isabella Invece di vendicare l'attentato commesso contro Flavio come aveva indicato all'inizio della commedia - Flaminia diventa la protettrice dei diritti di Isabella. 
si presenta a Ruggiero sotto la forma del mago Atlante ${ }^{10}$, vecchio precettore del paladino. Essendo un personaggio autoritario, l'apparizione di Atlante provoca una reazione di timore e di vergogna da parte di Ruggiero.

In questi due episodi, Flaminia e Melissa minacciano gli amanti per renderli ragionevoli. Benché i due personaggi femminili utilizzino mezzi diversi per arrivare alla meta - Melissa si serve della magia e della retorica, Flaminia invece usa la seduzione e la violenza - tutte e due le azioni sono coronate di successo. Nel canto XLVI dell' Orlando furioso, Ruggiero sposa Bradamante e nell'ultima scena de La pazzia d'Isabella Orazio prende Isabella in moglie e Flavio si unisce a Flaminia (O.f., XLVI, lxxiii e seguenti).

Un aspetto degno di attenzione sembra essere il fatto che l'atto violento di Isabella non venga giudicato dagli altri personaggi come atto di pazzia. È vero che Isabella dimostra anche una vena bellicosa durante la sua pazzia causata dall'amore infelice per Orazio. In una scena, Isabella "bastona il Capitano et Arlecchino, quali fuggono, et ella dietro seguitandoli” (Scala, La pazzia d'Isabella, III, p. 395). Questo atto è però caratterizzato da un aspetto burlesco, non comparabile con l'attentato d'omicidio contro Flavio. Ricordiamo che invece l'Orlando furioso dimostra la violenza di Orlando come gesto di follia, dovuto alla passione non ricambiata per Angelica ${ }^{11}$.

Lo stato squilibrato di Isabella, stato mentale a cui allude anche il titolo della commedia, non si manifesta però nel tentativo di uccidere Flavio, bensì nei discorsi incoerenti e talora scatologici della protagonista eponima.

Saturno vuol tirar una coreggia (...) e che per ciò fu veduto l'arco baleno far un serviziale all'Isola d'Inghilterra, che non poteva pisciare. (Scala, La pazzia d'sabella, III, p. 396.)

10 "Ne la forma d'Atlante se gli affaccia / colei, che la sembianza ne tenea, / con quella grave e venerabil faccia / che Ruggier sempre riverir solea, / con quello occhio pien d'ira e di minaccia, / che sì temuto già fanciullo avea." Ariosto, Orlando furioso, VII, lvi.

11 Nel canto XXXIV, Giovanni Evangelista spiega che la pazzia d'Orlando è stata una punizione divina dell'amore indegno del paladino per Angelica. "renduto ha il vostro Orlando al suo Signore / di tanti benefici iniquo merto; / che quanto aver piú lo dovea in favore, / n'è stato il fedel popul piú deserto. / Sí accecato l'avea l'incesto amore / d'una pagana, ch'avea già sofferto / due volte e piú venire empio e crudele, / per dar la morte al suo cugin fedele [Rinaldo] // E Dio per questo fa ch'egli va folle, / e mpostra nudo il ventre, il petto e il fianco; / e l'intelletto sí gli offuca e tolle, / che non può altrui conscere, e sé manco." O.f., XXXIV, lxiv-lxv. 
La volgarità del discorso di Isabella, accompagnata da gesti osceni, visto che l'attrice si spoglia sulla scena ${ }^{12}$, sembra poco conveniente al ruolo dell'innamorata. Ciononostante, un tale comportamento pare tipico delle rappresentazioni dell'Arte.

Anche lo strip tease, con buona pace dei moralisti antichi e recenti, rientra tra le "inventioni" del "recitar soggetti all'improvviso", facendo anch'esso parte di quell'adattabilità dello spettacolo al piacere pubblico di ogni sera: logica conseguenza dell'invenzione del teatro come merce, che è in fondo l'unico vero segreto della Commedia dell'Arte ${ }^{13}$.

Il tentativo d'omicidio commesso da Isabella resta senza conseguenze giuridiche. La minaccia espressa da parte del servo Pedrolino, secondo il quale Isabella sarebbe condannata a morte, è soltanto un gesto burlesco, mancando di serietà. Inoltre, Pedrolino specifica che Isabella non sarebbe l'unica ad essere punita, ma che Orazio dovrebbe pagare ugualmente il suo tradimento con la vita.

Pedrolino dice tra sé di voler burlar Pantalone [padre d'Orazio], al quale dice che Flavio è morto per le ferite che li dette Isabella, e che la giustizia la farà morire insieme con Orazio. (Scala, La pazzia d'Isabella, III, p. 395)

Essendo la minaccia di morte soltanto una burla da parte di Pedrolino, la commedia di Scala termina con un lieto fine. Isabella chiede scusa a Flavio, il quale la perdona subito ${ }^{14}$. E il reato di Flaminia contro Orazio resta pure senza conseguenze.

Per evidenziare l'originalità de La pazzia d'Isabella per quanto riguarda l'uso delle armi da parte dei personaggi femminili, oltre all'assenza di ogni condanna dei reati commessi da Isabella e da Flaminia, vorremmo comparare la commedia di Scala con la commedia Lo Schiavetto del drammaturgo e attore Giovan Battista Andreini (1576 o 1579-1654), figlio della famosa Isabella Andreini. Lo Schiavetto, commedia scritta nello stile della Commedia dell'arte, fu stampata per la prima volta nel 1612 .

Come La pazzia d'Isabella, anche la commedia di Andreini mette in scena un tentativo d'omicidio. La vittima è ancora una volta l'amante infedele. A confronto con la commedia di Scala, la vendetta è commessa diret-

\section{Marotti/Romei, La commedia dell'arte, XLVI-XLVII.}

14 "Flavio col braccio al collo; vede Isabella, la quale umilmente li chiede perdono, facendoli sapere come Orazio l'ha sposata di fede. Flavio si rallegra e li perdona." Scala, La pazzia d'Isabella, III, p. 396. 
tamente dall'amata tradita, Florinda, che si traveste da "schiavetto" per vendicarsi dell'infedeltà di Orazio.

Sappiate adunque che una donna detta Florinda, che in Napoli tra Orazio e lei passò fede di matrimonio e godimento, è quella che in abito di schiavo e sotto nome apposito di Schiavetto, schiava di fortuna andava errando, per far tanto schiavo di morte Orazio, quanto Orazio aveva di lei fatta schiava del disonore. (...) Orazio di Florinda (ch'è l'ingannata) s'era dimenticato, per ricordarsi di Prudenza, tanto lo sdegno la [Florinda] dominò, che più pronta all'odiare che all'amare, s'appigliò come sdegnata, come infuriata,di vendicarsi, ma non contro di te [Prudenza], ma contro di colui che in istato così misero posta l'avea ${ }^{15}$.

Per castigare il tradimento commesso da Orazio, Florinda non ha ricorso alle armi, ma al veleno. Questo metodo sembra essere iscritto nella tradizione letteraria come arma prediletta della vendetta femminile ${ }^{16}$. Anche l' Orlando furioso ci fornisce un esempio. L'omicidio di Tanacro, raccontato nel canto XXXVII dell'epopea ariostesca, riprende la famosa storia di Camma, narrata tra l'altro da Plutarco nel suo trattato sopra la virtù delle donne ${ }^{17}$. Sinorige, principe di Galizia è innamorato di Camma, moglie di Sinato. Per poter sposare Camma, Sinorige decide di uccidere Sinato. Camma, fingendo di accettare lo sposalizio con Sinorige, lo avvelena durante la cerimonia di nozze nel tempio di Diana. L'Ariosto aveva adattato questo mito, trasponendolo sui personaggi di Tanacro (Sinorige) e di Drusilla (Camma). Questo episodio fa parte dei passaggi filogini del poema ariostesco, dal momento in cui vengono castigati sia il reato di Sinorige sia quello di suo padre Marganorre. Costui mise in vigore delle leggi misogine per punire la morte del figlio. Grazie alla prodezza di Marfisa e di Bradamante, Marganorre paga il suo odio verso il sesso femminile con la vita ${ }^{18}$. Il lato filogino di questo episodio sembra aver ispirato l'attrice e drammaturga Flaminia Romana che nell'estate del 1567 mise in scena la storia di Drusilla e di Tanacro. Purtroppo il canovaccio della sua tragedia è smarrito, tuttavia esiste la testimonianza di uno spettatore. Si tratta di Luigi Rogna, segretario alla corte dei Gonzaga, che in una lettera datata del 6 luglio 1567 scrive:

15 Andreini, Lo Schiavetto, V.vi, p. 179.

16 Schmal, "Frauen und Barbaren bei Euripides", pp. 97-98.

17 Plutarco, "Mulierum virtutes", pp. 551-555.

18 Per un'analisi interessante di quest'episodio, riferiamo al saggio di Bellamy, "Androgyny and the Epic Quest" e a Benson. The Invention of the Renaissance Woman. 
Non hieri l'altro la Flaminia era comendata per certi lamenti che fece in una tragedia che recitorno dalla sua banda, cavata da quella novella dell'Ariosto, che tratta di quel Marganorre, al figliuolo sposo del quale, la sposa, ch'era Flaminia, sopra il corpo del primo suo sposo, poco dianzi amazzato in scena, per vendetta diede a bere il veleno dopo haverna bevuto anch'essa, onde l'uno et l'altro morì sopra quel corpo, et il padre, che perciò voleva uccidere tutte le donne, fu dalle donne lapidato a morte ${ }^{19}$.

Se Flaminia sembra aver cambiato la fine dell'episodio ariostesco, tralasciando l'intervento armato di Marfisa e Bradamante, il carattere dell'attrice allude nondimeno alle virago ariostesche. In una rissa avvenuta a Mantova nel 1566 tra i comici della truppa di Flaminia e i soldati di Cesare Gonzaga, Flaminia intercede coraggiosamente, facendo cessare la lite.

Alle 22 hore nel accompagnar alla sepoltura mio fratello al carmine, si stacchi una delle maggiori questioni, che mai m'habbia veduto, per il numero grandissimo dell'armi men[d]ate; la quale era tra li comedianti et quelli del Signor Cesa Illustrissimo, et la Flaminia fecce con gli sassi cose stupende, al pari d'una Marfisa, et se lei non era senza dubbio, ne ne riniaverrano morti ${ }^{20}$.

Essendo comparata alla virago ariostesca e avendo adattato un passaggio filogino dell' Orlando furioso, l'intenzione di Flaminia sembra chiara: provocare il pubblico e mettere in dubbio la gerarchia dei sessi.

To some degree the actress [Flaminia Romana ] plays herself, and as she redoubles the process of masking and unmasking feminine roles for a male audience, she alters the comic exposure of female masquerade that Ariosto develops but contains within the 'carte' of his book. If, as Valeria Finucci proposes, the cross-dressed, bigendered Marfisa pays a severe narrative price for performing such exposure on the page (Finucci, "Female Masquerade", 80-81), the Marfisa-like Flaminia-as-Drusilla becomes a hero by enacting it on the stage. The live performance of this episode entertained its spectators, but it also may have challenged them. For in this case, they saw a woman in a variety of guises, who performs the masquerade of femininity only to undo it, and who revises Ariosto by directing her company to kill Marganorre before he can kill any of the women: in this female revision, the misogynist's law and disorder is not at all enforced, let alone written. ${ }^{21}$

19 D'Ancona, Origini del teatro italiano, 2:451.

20 Nicholson, "Romance as Role Model”, 246.

21 Nicholson, "Romance as Role Model”, 262. 
Tenendo conto del fatto che l'avvelenamento è una delle armi "classiche" femminili e che Scala conosceva il mito di Camma e la sua versione ariostesca, la presa d'armi di Isabella e di Flaminia, il cui personaggio allude forse anche all'attrice eponima Flaminia Romana, rafforza l'originalità della commedia La pazzia d'Isabella.

A parte la vendetta della donna tradita, Lo Schiavetto e La pazzia d'Isabella mostrano un altro punto in comune. Tutte e due le protagoniste, Isabella e Florinda, ammettono in pubblico il loro reato ${ }^{22}$. Tuttavia, Florinda non dà nessuna spiegazione riguardante la sua identità o il motivo dell'avvelenamento.

Sappiasi che per commissione d'un suo capitalissimo nemico sono andato gran tempo cercando costui, per tôrgli la vita; e oggi appunto con que' moscardini hollo avelenato. (Andreini, Lo Schiavetto, IV.ix, p. 165)

Contrariamente alla commedia di Scala, nella quale gli atti di violenza di Isabella e di Flaminia non sono commentati da parte dei dramatis personae, il tentativo d'omicidio di Florinda viene criticato. I personaggi che circondano Florinda, la insultano chiamandola "Ah traditore", "Ah crudo avelenatore", "Ah furbaccio" (Andreini, Lo Schiavetto, IV.ix, p. 165).

Nella commedia di Andreini, il bargello interviene immediatamente. La scoperta che lo "schiavetto" è in realtà una donna non cambia la punizione di Florinda, condannata a morte ${ }^{23}$. Soltanto dopo aver spiegato le ragioni che hanno indotto Florinda a voler uccidere Orazio, lo "schiavetto" è graziato. Secondo la morale della commedia, la punizione di un amante infedele sembra essere una legittima difesa. Il tono farsesco con cui viene descritta la grazia di Florinda, sottolinea però l'aspetto ironico di questa scena.

In questo mentre, "Grazia, grazia!" tutto il popolo chiamando, alle voci di tanti commosso, il signore grazie disse, e grazia le [Florinda] fece. (Andreini, Lo Schiavetto, V.vi, p. 180)

Questa scena allude probabilmente al personaggio della virgo bellatrix che incarna nelle stampe della seconda metà del Cinquecento un nuovo ideale femminile: da vittima fragile, la donna si trasforma in guerriera

22 "Flavio cade in terra versando il sangue; in quello Orazio arriva. Isabella, abbracciandolo e dicendoli d'aver fatte le sue vendetto, lo conduce in casa." Scala, La pazzia d'Isabella, 392.

23 "questo Schiavetto mentre andava inanzi al signor governatore, udendola (ben che scoperta donna) condannarla a morte.” Andreini, Lo Schiavetto, V.vi, p. 180. 
robusta che protegge la sua castità e il suo corpo senza aver bisogno di un protettore maschile 24 . Il fatto che Florinda venga graziata, mette in rilievo l'importanza dell'auto-protezione femminile del proprio corpo. La commedia di Andreini riprende quest'immagine della virgo bellatrix, però in maniera caricaturale. In primo luogo, Florinda non è più una virgo, visto che ad Orazio "la prima rosa coglier lasciò" (O.f., XIX, xxxiii, 1-2), secondo l'espressione ariostesca. Inoltre, Lo Schiavetto mette in evidenza, con un tono burlesco, l'importanza della virtù femminile, ponendola al di sopra della vita umana. Avendo abbandonato Florinda dopo le nozze, Orazio è degno di morte. L'intenzione parodistica dell'autore sembra palese.

All'aspetto buffonesco della commedia di Andreini bisogna aggiungere anche un tono misogino. Malgrado il lamento di Florinda indirizzato al suo amante infedele, la donna gli concede allo stesso tempo il potere di giudicare il suo tentativo d'omicidio. Pur essendo graziata dalla giustizia, Florinda si sottopone all'indulgenza di Orazio, che nella scena precedente voleva ancora uccidere.

Però eccomi a’ piedi tuoi, se di vita e di mercede mi giudichi non indegna, dammi mercede e vita ; e se morta mi vuoi, di' solo muori (...). (Andreini, Lo Schiavetto, V.viii, p. 186)

Questo atteggiamento da parte di Florinda ricorda l'immagine della donna ideale nei trattati di Sperone Speroni che nel Dialogo della dignità delle donne (1543) fa l'elogio della sommissione femminile.

Serva adunque la Donna, perchè a servire è creata, ma non l'aggravi tal servitù consciosiacosa ch'ella non serve si come priva di libertà et a guisa di schiava, ma come cose, cui l'esser Libera tanto, ò quanto non si convenga; mancando per sua natura, di quella parte dell'anima, onde è dato a voi huomini che voi debbiate signoreggiarne. (Speroni, "Della Dignità delle Donne", 62.)

Il carattere sommesso di Florinda contraddice la sua precedente rappresentazione da virgo bellatrix che prende in mano il suo proprio destino. Contrariamente al ritratto femminile dell'opera di Sperone Speroni, l'immagine della donna ideale ne Della nobiltà et eccellenza delle donne et $i$ difetti, e mancamenti de gli uomini (1600) di Lucrezia Marinella celebra l'individualità e la libertà femminile che ritroviamo anche ne La Pazzia d'Isabella. Secondo Marinella, sono le donne che governano il mondo e che signoreggiano gli uomini, a loro inferiori. 
Onde si potrebbe dire (...) che la donna produca il poco cortese maschio, li dia anima, \& vita, lo illumini con lo splendor della divina luce; lo conservi in questa terrena spoglia coil calora $\&$ cola luce, lo renda al contrario delle fiere d'animo affabile, \& cortese, \& finalmente lo signoreggi con un dolce $\&$ non punto tirannico impero. (Marinella, Della nobiltà et eccellenza delle donne, 3)

La commedia di Scala sembra corrispondere a certe idee di Lucrezia Marinella, facendo l'elogio di un'autorità femminile: non è l'amante infedele ad accordare il suo perdono, ma Isabella, la donna tradita.

Ritornata saggia, [Isabella] vede Orazio, al quale ricorda quanto ha fatto per lui, con breve giro di parole, lamentandosi che l'abbia tradita e per altra donna abbandonata. Orazio confessa l'error suo et il suo mancamento, li chiede perdono, dicendoli volerla sposare allora allora. Isabella, tutta allegra, pone in oblìo ogni passata cosa e l'accetta per suo. (Scala, La pazzia d'Isabella, III, p. 396)

Comparando la commedia di Scala con quella di Andreini, constatiamo che La pazzia d'Isabella si distingue per la sua originalità e per un tono filogino. I reati di Isabella e di Flaminia non hanno bisogno di essere giustificati, contrariamente alla commedia di Giovan Battista Andreini. Le due protagoniste de La pazzia d'Isabella agiscono come se la presa delle armi fosse cosa corrente e atta al sesso femminile. I gesti di Isabella e di Flaminia non sono considerati come atti di trasgressione, ma rappresentano un ritorno all'ordine. Soltanto dopo esser stato aggredito da Flaminia, Orazio ritrova la sua ragione e rimpiange il fatto di aver tradito Isabella ${ }^{25}$. I gesti violenti di Flaminia e di Isabella attribuiscono perciò al consolidamento della norma: la commedia finisce con un doppio matrimonio e con un rinforzamento della stabilità. Il tono filogino de La pazzia d'Isabella sembra suggerire che la stabilità ritrovata non esclude però uno spazio d'azione concesso ai personaggi femminili. Nelle commedie di Scala, le donne trovano sempre un modo per proteggere i loro interessi.

Un certo rinvigorimento dell'ordine si ritrova anche nella commedia di Andreini. Il rafforzamento della norma non dipende però dall'attentato di Florinda, bensì dal perdono e dall'indulgenza di Orazio. L'ordine restaurato nella commedia di Andreini equivale quindi ad un consolidamento della gerarchia tra i sessi. Ciò dimostra anche la fine della commedia che si con-

25 Linfedeltà di Orazio potrebbe simboleggiare una seconda forma di "pazzia", a parte quella di Isabella. Il comportamento dell'amante incostante trasgredisce le norme sociali. 
clude non solo con il matrimonio tra Orazio e Florinda, ma anche tra Prudenza, rivale di Florinda, e Fulgenzio. Quest'ultimo, essendo ripudiato da Prudenza, che gli preferisce Orazio, decide di violentarla, sperando di poterla sposare. Ci sembra degno di attenzione che Prudenza, in un primo momento di furia, voglia uccidere il suo stupratore con un coltello. Ciò rappresenta un altro punto in comune con le eroine della commedia di Scala. Ma contrariamente a Flaminia e ad Isabella, Prudenza non fa uso della sua arma, dichiarando che:

... è meglio che donna rimanga più tosto onorata e invendicata, che vendicata e disonorata, Fulgenzio, pace trovando ne' miei interni contrasti, io ti perdono. (Andreini, Lo Schiavetto, V.vi, p. 177)

Lo sposalizio di Prudenza e Fulgenzio dipende, come anche quello di Orazio e Florinda, dal perdono del traditore, in questo caso di Prudenza. All'opposto di Orazio, che, vinto dall'amore di Florinda, si decide di prenderla in moglie, Prudenza non è tanto condizionata dal sentimento quanto dalla morale in vigore, simboleggiata da Imeneo, dio delle nozze.

Ecco Imeneo che fa il colpo arrestar così dicendomi: "Che farai micidiale? Al fine dura forza ti sforza a chiamarlo consorte, e come tale bramar anche la salute sua..." (Andreini, Lo Schiavetto, V.vi, p. 176).

Se Florinda riesce a convincere Orazio di sposarla con gesti di umiltà, dissimulando la sua persona sotto i panni di un servo, Fulgenzio al contrario, conquista il cuore di Prudenza tramite la "dolce violenza" (Andreini, Lo Schiavetto, V.vi, p. 177).

In conclusione possiamo constatare che il rapporto tra La pazzia d'Isabella e Lo Schiavetto rispetto alla Querelle des femmes è caratterizzato dal tono filogino dell'una e misogino dell'altra. La rappresentazione positiva delle due protagoniste de La pazzia d'sabella sottolinea una certa intraprendenza femminile e l'importanza di una partecipazione della donna nell'ambito politico-sociale, visto che il ritorno alla norma si fa grazie alle due protagoniste. La commedia di Andreini mette invece in luce il potere maschile. Ne Lo Schiavetto, la possibilità d'azione di Florinda è limitata. Per arrivare al suo fine - le nozze con Orazio - Florinda deve essere graziata sia dal governatore, che simboleggia la giustizia, sia dal suo amante. Entrambi personificano il potere delle istituzioni patriarcali.

Una certa insofferenza da parte di Andreini verso l'intraprendenza femminile si dimostra anche al di là delle sue opere letterarie. In una lettera indirizzata a Ferdinando Gonzaga, scritta a Milano il 12 giugno 1609, Andreini si lamenta dell'attrice Orsola Posmoni Cecchini, chiamandola 
"Marfisa bizarra".

Questa discordia (fra le altre molte) è pur cagionata ancora perché mi danno quella poca parte (...)et haverà ottenuto il poeta [Pier Maria Cecchini, attore della compagnia rivale Gli Accesi] l'intento suo, (...) in andare a Genova a compiacenza della Marfisa bizarra. (Comici dell'Arte, 82)

L'editore della raccolta delle lettere di Andreini precisa che il termine "Marfisa bizarra" ha "un significato negativo di donna aggressiva e impudente" 26 . Inoltre, quest'espressione si riferisce probabilmente all'epopea eponima di Giovanni Battista Drangoncino, pubblicata per la prima volta nel $1531^{27}$. In quest'opera, Dragoncino rappresenta l'eroina ariostesca in preda al dominio della follia. Marfisa, seguendo l'esempio cattivo di Orlando, s'innamora di un giovane paladino senza essere ricambiata.

Ma poi ch'el Conte [Orlando] l'alma al tempo rese

De l'amorose sue fatiche tante,

Che lungo tempo sopportò in palese,

Per Angelica bella di Levante

Marfisa quel che già in altri risplende,

Le vestigie imitò di quel d'Anglante [Orlando]

In questo amor dice Turpin che il narra,

Che Marfisa tenuta fu bizarra.

Non vò dir, che venisse in quel furore

Ch'altro non canto di quel degno Conte [Orlando] $\cdots$

Ma ben Marfisa in questo estremo ardore

Entra in cieco desio sfrenato e sciolto.

Poca fiamma di donna salda il core,

Dentro a begl'occhi d'un leggiadro volto.

(Dragoncino da Fano, Marfisa bizarra, I, p. 4)

Dragoncino sottolinea una differenza netta tra il conte Orlando, degno paladino, e la donna guerriera Marfisa. Mentre Orlando, pur aman-

\section{Comici dell'arte, 84 .}

27 A partire dalla fine del Seicento, il termine "Marfisa bizarra" diventa un'espressione corrente, come dimostra anche il capitolo De' cervelazzi terribili, indomiti, diavolosi, intraversati, precipitosi, trapanati, bizzarri, bislacchi, balzani ed eterocliti nell'opera di Tomaso Garzoni. "Il proprio loro [cervelazzi diabolici] è d'andar sulla gamba come gradassi ; guardar col viso bieco come Orlandi ; fulminar di colera come Mandricardi ; esser bizzarri come Marfisa", Tomaso Garzoni, "Il teatro de'vari e diversi cervelli mondani”, 235. 
do Angelica, non sarebbe mai stato vittima della pazzia, Marfisa invece sarebbe diventata bizarra per amore. Ora i lettori dell'Ariosto sanno benissimo che l'eroe eponimo rischiò non solo di perdere la ragione a causa del suo amore per Angelica, ma anche la sua riputazione. Il titolo dell'epopea ariostesca lo dichiara apertamente. Marfisa però, essendo donna, è esposta più facilmente alla passione. Il carattere incostante del sesso femminile è evidenziato nell'opera di Dragoncino. Ricordiamo, che la parola "bizarra" non significa solo "stravagante", ma anche "violento"28. La "Marfisa bizarra" è perciò un personaggio furioso ed eccentrico, in altre parole, una "donna combattiva" 29.

Quindi non ci sembra sorprendente che Andreini non abbia scelto una donna guerriera come protagonista della sua commedia, dal momento che questo personaggio può essere connotato in maniera negativa. In effetti, Andreini sembra aver trovato un certo piacere ad evocare l'eroina di Dragoncino, visto che ne allude anche ne Lo schiavetto. La rivale di Florinda, la bella Prudenza, rifiuta all'inizio della commedia di far entrare in casa Nottola, un falso conte. Costui la insulta nel modo seguente:

Alberto, chi è questa giovinaccia, anzi questa Marfisa bizarra? (Andreini, Lo Schiavetto, I.viii, p. 95)

La diffidenza di Andreini verso l'intraprendenza femminile è sorprendente se si pensa al fatto che la madre dell'autore, Isabella Andreini, fu ugualmente un personaggio molto attivo nel mondo dello spettacolo e nel mondo letterario. Nel 1589, Isabella Andreini recitò alla corte dei Medici in una commedia con lo stesso titolo, però con un contenuto diverso da $\mathrm{La}$ pazzia d'Isabella di Scala. Esiste nondimeno un punto in comune tra queste due commedie: la pazzia della protagonista causata da un amore infelice. Purtroppo il canovaccio della commedia di Isabella Andreini è andato perso. Ci resta tuttavia la descrizione da parte di uno spettatore che assistette alla rappresentazione. L'interpretazione di Isabella mette in scena un personaggio in preda alla follia.

... così vinta dalla passione e lasciandosi superare alla rabbia, \& al furore uscì fuori di se stessa, \& come pazza se n'andava scorrendo per la Cittade, fermando hor questo, \& hora quello, e parlando hora in Spagnuolo, hora in Greco, hora in Italiano, \& molti altri linguaggi, ma

28 Dictionnaire Etymologique du Français, 51.

29 "Marfisa bizarra: personaggio dell'Ariosto, sinonimo di donna combattiva" (Falavolti in Andreini, Lo Schiavetto, I, 95). 
tutti fuori proposito $\ldots{ }^{30}$

Il riferimento ai sentimenti della "rabbia" e del "furore" espressi dalla protagonista, ricordano il personaggio della "Marfisa bizarra". L'eroina interpretata da Isabella Andreini, descritta dal figlio Giovan Battista Andreini come "sempre alla vertute intenta" 31 , avrà forse più in comune con l'eroina del Dragoncino, anche lei impazzita per un amore infelice, di quanto se ne possa immaginare. Inoltre, l'interpretazione strip tease data da Isabella Andreini quando recitò la parte della protagonista eponima nella commedia di Scala, non corrisponde del tutto all'immagine celebrata dal figlio. Come indicano Marotti e Romei, la virtù di Isabella Andreini è soltanto uno degli aspetti molteplici dell'attrice.

Se da un lato la descrizione di Isabella in azione non smentisce tanto la fama della sua dottrina e della sua compostezza espressiva ..., dall'altro svela un aspetto inedito dell'attrice, non tanto la sua proteiformità, quanto la sua capacità di interpretare "parti ridicole", di uscire cioè dal proprio ruolo $\ldots{ }^{32}$

Aggiungiamo che le "parti ridicole" interpretate da Isabella Andreini contengono anche delle "parti erotiche", basti pensare allo "spogliarello" effettuato sulla scena.

Se Andreini dipinge la donna guerriera sotto una luce negativa, Scala la rappresenta in maniera positiva. Flaminia e Isabella non vengono trattate da "Marfise bizarre". Tutt'altro, sono loro a far ritornare l'ordine, dopo il disordine provocato dall'infedeltà di Orazio. Anche in ciò le protagoniste de La pazzia d'Isabella assomigliano alle guerriere ariostesche che, secondo la ricercatrice Chimène Bateman, rappresentano i pilastri del Orlando furioso.

Whereas the Amazon of antiquity, in keeping with the Greek tendency to conceptualize difference in terms of polarity, is largely defined as what the Greeks themselves are not, Ariosto's guerriere play a new role: not only are they central to the plot, but they stand as emblems of it. The hybrid figure of the woman warrior, monstruos within the ancient world, is relocated to a universe where hybridity is the norm. ${ }^{33}$

30 Marotti/Romei, La commedia dell'arte e la società barocca, lxxv.

31 Andreini, "La Ferza”, 497.

32 Marotti/Romei, La commedia dell'arte e la società barocca, 165.

33 Bateman, "Amazonian Knots”, 21. 
Flaminia e Isabella incarnano perciò un ideale femminile che si colloca tra la prodezza virile e la fedeltà e la lealtà dell'amata verso il suo amante. In più, le protagoniste di Scala simboleggiano una forma teatrale che trova la sua dinamica e la sua originalità nell'improvvisazione che non solo lascia spazio ad un nuovo modo di recitare, comparato con il teatro prima della Commedia dell'arte, ma anche ad una nuova libertà femminile. Siccome Giovan Battista Andreini appartiene alla generazione successiva di Flaminio Scala, Lo Schiavetto mette in luce il declino dell'immagine della guerriera che caratterizzerà il Seicento e il Settecento. Il personaggio della virago non avrà più il suo posto nella commedia bensì nell'opera lirica $\mathrm{e}$ nell'opera buffa.

\section{PARIS VII DENIS DideroT}

\section{OPERE CITATE}

Andreini, Giovan Battista. "La Ferza. Ragionamento secondo. Contra l'accuse date alla Commedia." In Ferruccio Marotti e Giovanna Romei, La commedia dell'arte e la società barocca. Roma: Bulzoni Editore, 1994, pp. 489-534.

. Lo Schiavetto. In Commedie dei comici dell'arte, a c. di Laura Falavolti. Torino: UTET, 1982, pp. 63-208.

Ariosto, Ludovico. Orlando furioso. Torino: Giulio Einaudi, [1532] 1966.

Bateman, Chimène. "Gender, Genre, and Ariosto's Women Warriors”. MLN, 122 (2007): 1-24.

Comici dell'arte. Corrispondenze. G.B. Andreini, N. Barbieri, P.M. Cecchini, S. Fiorillo, T. Martinelli, F. Scala. a c. di Siro Ferrone. Firenze: Casa Editrice Le Lettere, 1993.

D’Ancona, Alessandro. Origini del teatro italiano. 2 voll. Roma: Bardi Editore, [1891], 1971.

Dictionnaire Etymologique du Français. Parigi: coll. Les Usuels du Robert Poche, Dictionnaires le Robert, 2002.

Dragoncino da Fano, Giovanni Battista. Marfisa bizzarra. Padova: Per Gio: Antonio Remondini, s.d.

Garzoni, Tomaso. "Il teatro de' vari e diversi cervelli mondani." In Opere, a c. di Paolo Cerchi. Ravenna: Longo Editore Ravenna, 1993, pp. 47-243.

Locatelli, Basilio. "Orlando Furioso." In Vito Pandolfi, La commedia dell'arte. Storia e testo, Firenze: Casa Editrice Le Lettere, 1988, vol. 5, pp. 236-237.

Marinella, Lucrezia. Della nobiltà et eccellenza delle donne et $i$ difetti, e mancamenti de gli uomini. Venetia: Appresso Giovan Battista Ciotti Senese, 1600.

Mathews, Sara F. Ange ou Diablesse. La représentation de la femme au XVIe siècle. Paris: Flammarion, 1991.

Plutarco. "Mulierum virtutes." In Moralia, a c. di Frank Cole Babbitt. Cambridge, MA: Harvard University Press, [1931], 1989,vol. 3, pp. 551-555. 
Nicholson, Eric. "Romance as Role Model: Early Female Performances of Orlando furioso and Gerusalemme liberata." In Renaissance Transactions. Ariosto and Tasso, a c. di Valeria Finucci. Durham and London: Duke University Press, 1999, pp. 246-269.

Scala, Flaminio. Le disgrazie di Flavio. In Ferruccio Marotti, Il teatro delle favole rappresentative. 2 voll. Milano: Edizioni Il Polifilo, 1976, vol.2, pp. 353-362.

- La pazzia d'Isabella. In Ferruccio Marotti, Il teatro delle favole rappresentative, Milano: Edizioni Il Polifilo, 1976, pp. 385-396.

. "Prologo per recitare." In Ferruccio Marotti e Giovanna Romei, La commedia dell'arte e la società barocca. La professione del teatro. Roma: Bulzoni Editore, 1994, pp. 552-554.

Schmal Stephan. "Frauen und Barbaren bei Euripides." In Geschlechterrollen und Frauenbild in der Perspektive antiker Autoren, a c. di Robert Rollinger e Christoph Ulf. Innsbruck: Studienverlag, 2000, pp. 87-128.

Tylus, Jane. "Women at the Windows: Commedia dell'arte and Theatrical Practice in Early Modern Italy." Theatre Journal 49.3 (1997): 323-342.

Zimmermann, Margarete. "Vom Streit der Geschlechter. Die französische und italienische Querelle des Femmes des 15. und 17. Jahrhunderts." In Die Galerie der starken Frauen. Die Heldin in der französischen und italienischen Kunst des 17. Jahrbunderts. Ausstellungskatalog, a c. di Bettina Baumgärtel e Silvia Neysters. Berlin, München: Beilharz \& Klinkmann, 1995, pp. 14-33. 